\title{
Inhibition of HIV transmission in human cervicovaginal explants and humanized mice using CD4 aptamer-siRNA chimeras
}

\author{
Lee Adam Wheeler, ${ }^{1,2}$ Radiana Trifonova, ${ }^{1}$ Vladimir Vrbanac, ${ }^{3}$ Emre Basar, ${ }^{1}$ Shannon McKernan, ${ }^{1}$ \\ Zhan Xu, ${ }^{1}$ Edward Seung, ${ }^{3}$ Maud Deruaz, ${ }^{3}$ Tim Dudek, ${ }^{4}$ Jon Ivar Einarsson, ${ }^{5}$ Linda Yang, ${ }^{6}$ \\ Todd M. Allen, ${ }^{4}$ Andrew D. Luster, ${ }^{3}$ Andrew M. Tager, ${ }^{3}$ Derek M. Dykxhoorn,, ${ }^{17}$ and Judy Lieberman ${ }^{1}$ \\ ${ }^{1}$ Immune Disease Institute and Program in Cellular and Molecular Medicine, Children's Hospital Boston, Harvard Medical School, Boston, Massachusetts, USA. \\ 2MD-PhD Program (Immunology), Harvard Medical School, Boston, Massachusetts, USA. ${ }^{3}$ Massachusetts General Hospital, Boston, Massachusetts, USA. \\ ${ }^{4}$ Ragon Institute of MGH, MIT, and Harvard, Boston, Massachusetts, USA. ${ }^{5}$ Brigham and Women's Hospital, Boston, Massachusetts, USA. \\ ${ }^{6}$ Beth Israel Deaconess Medical Center, Boston, Massachusetts, USA. 7 University of Miami Miller School of Medicine, Miami, Florida, USA
}

\begin{abstract}
The continued spread of the HIV epidemic underscores the need to interrupt transmission. One attractive strategy is a topical vaginal microbicide. Sexual transmission of herpes simplex virus type 2 (HSV-2) in mice can be inhibited by intravaginal siRNA application. To overcome the challenges of knocking down gene expression in immune cells susceptible to HIV infection, we used chimeric RNAs composed of an aptamer fused to an siRNA for targeted gene knockdown in cells bearing an aptamer-binding receptor. Here, we showed that CD4 aptamer-siRNA chimeras (CD4-AsiCs) specifically suppress gene expression in $\mathrm{CD}^{+} \mathrm{T}$ cells and macrophages in vitro, in polarized cervicovaginal tissue explants, and in the female genital tract of humanized mice. CD4-AsiCs do not activate lymphocytes or stimulate innate immunity. CD4-AsiCs that knock down HIV genes and/or CCR5 inhibited HIV infection in vitro and in tissue explants. When applied intravaginally to humanized mice, CD4-AsiCs protected against HIV vaginal transmission. Thus, CD4-AsiCs could be used as the active ingredient of a microbicide to prevent HIV sexual transmission.
\end{abstract}

\section{Introduction}

The CAPRISA004 study, which demonstrated partial protection from sexual transmission of HIV-1 by vaginally applied tenofovir gel (1), has galvanized interest in developing an HIV microbicide. One of the major obstacles confronting this and other strategies for interrupting transmission is the transience of protection, requiring topical application just before sexual exposure and raising associated problems with compliance (2). Soon after RNAi was found to operate in mammalian cells, multiple groups showed that RNAi could be harnessed to inhibit HIV infection in vitro (3-7). Moreover, siRNAs directed against conserved viral gene sequences or the HIV receptor or coreceptor inhibit diverse viruses from multiple clades $(3,8,9)$. Although knocking down the HIV receptor $C D 4$ inhibits HIV transmission (3), targeting the $C D 4$ gene would likely interfere with mounting effective immune responses and is therefore not desirable. CCR5, the HIV coreceptor responsible for virtually all sexual transmission of $\operatorname{HIV}(10,11)$, is a more attractive RNAi target. CCR5 antagonists (12) have already proven useful at preventing HIV transmission in nonhuman primates (13) and humans (14-17). Humans bearing homozygous CCR5 mutations that abrogate CCR5 function are resistant to HIV infection and do not lead to any significant immune dysfunction (18-22). siRNAs directed against CCR5 efficiently silence gene expression for several weeks in vitro in nondividing macrophages, which suggests that gene knockdown might be used to induce durable resistance to HIV infection, circumventing the need to apply a microbicide just before each sexual encounter (8). In fact, sexual transmission of another virus, herpes simplex

Conflict of interest: The authors have applied for a patent based on this work. Citation for this article: J Clin Invest. 2011;121(6):2401-2412. doi:10.1172/JCI45876. virus type 2 (HSV-2), can be blocked in mice for at least a week by intravaginal (IVAG) application of siRNAs targeting HSV-2 genes and the HSV-2 receptor, nectin-1 $(23,24)$.

Translation of these promising results for blocking HSV-2 transmission to HIV prevention, however, must first overcome the hurdle of in vivo siRNA delivery to the immune cells that HIV infects, principally $\mathrm{CD} 4^{+} \mathrm{T}$ cells and macrophages, which are resistant to most transfection techniques. Although cholesterol-conjugated siRNAs are efficiently taken up by epithelial cells throughout the genital tract (including deep in the lamina propria, resulting in protection against lethal HSV-2 infection in mice; ref. 24), these reagents do not knock down gene expression in $\mathrm{T}$ lymphocytes or macrophages when applied IVAG to mice (E. Basar, unpublished observations). We previously developed a method for cell-specific siRNA transfection of immune cells that uses a fusion protein composed of a cell-targeting antibody fragment joined to a protamine peptide that binds nucleic acids $(25,26)$. siRNAs mixed with the fusion protein are taken up by and knock down gene expression in cells bearing the cognate surface receptor, both in vitro and in tissues after intravenous injection. Modifications of this approach effectively inhibit HIV infection in humanized mice (27). However, antibodybased fusion proteins are expensive to manufacture, are potentially immunogenic, and may require refrigerated storage, making then ill-suited for use in a microbicide for resource-poor settings.

Chimeric RNAs, composed of an siRNA fused to an aptamer (a structured RNA selected to bind a cell surface ligand with high affinity), provide an attractive alternative for in vivo gene knockdown (28-31). Aptamer-siRNA chimeras (AsiCs) efficiently transfect and knock down gene expression in cells bearing the surface receptor recognized by the aptamer. Intravenous injection of AsiCs incorporating aptamers targeting prostate surface membrane $\mathrm{Ag}$ 

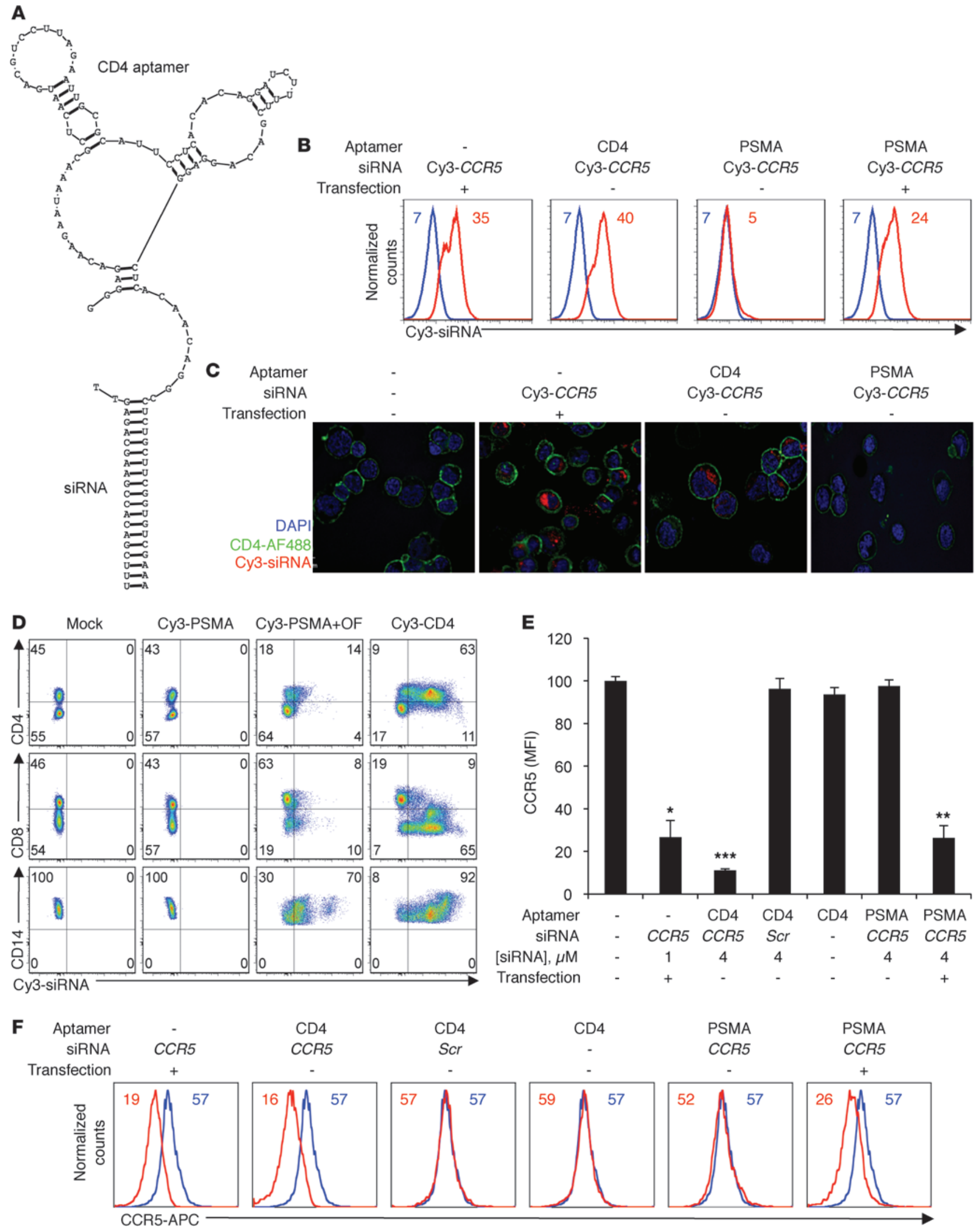


\begin{abstract}
Figure 1
Cy3-labeled CD4-AsiCs are internalized by $\mathrm{CD} 4{ }^{+}$cells and silence CCR5 expression in vitro. (A) Design of CD4-AsiC, containing a CD4 aptamer and a CCR5 siRNA. (B and C) CD4-AsiCs or PSMA-AsiCs targeting CCR5 were Cy3 labeled at the $3^{\prime}$ end of the antisense siRNA strand and incubated with primary $\mathrm{CD} 4^{+} \mathrm{T}$ lymphocytes from a healthy donor. Uptake was assessed 24 hours later by flow cytometry (B) and fluorescence microscopy (C; original magnification, $\times 60$ ). Data are representative of 3 independent experiments. MFI of each peak is shown (mock, blue; treated, red). Transfection controls used nucleofection. (D) Specific siRNA delivery to CD4+ cells in a mixed population of resting PBMCs was assessed by flow cytometry 24 hours after incubation with $4 \mu \mathrm{M}$ Cy3-labeled AsiCs. In the absence of oligofectamine (OF), Cy3-labeled CD4-AsiCs were preferentially taken up by $C D 3^{+} C D 4^{+}$ $\mathrm{T}$ cells and $\mathrm{CD} 4^{+} \mathrm{CD} 14^{+}$monocytes, whereas PSMA-AsiCs only transfected monocytes with OF. $\mathrm{CD} 3^{+} \mathrm{CD} 8^{+} \mathrm{T}$ cells remained relatively label free. Representative dot plots of 3 experiments with different donors are shown. ( $E$ and $\mathbf{F}$ ) To test for gene silencing, CD4+ $T$ lymphocytes were treated with CD4-AsiCs or PSMA-AsiCs targeting CCR5, with or without transfection. Chimeras bearing scrambled siRNAs (Scr) and CD4 aptamers served as controls. Shown are mean \pm SEM MFI normalized to the mock-treated sample $\left(\mathrm{E} ; n=4\right.$; ${ }^{*} P<0.005$, ${ }^{* \star} P<0.0005,{ }^{* \star *} P<0.00005,2$-tailed $t$ test) and representative histograms (F; mock, blue; treated, red). In the absence of nucleofection, CCR5 was knocked down only by CCR5 CD4-AsiCs.
\end{abstract}

(PSMA) silence target gene expression in orthotopic prostate cancer mouse xenografts $(28,29)$. AsiCs containing an aptamer that recognizes HIV-gp120 inhibit HIV replication in already infected cells in vitro $(30,31)$ and in vivo $(32)$. However, to prevent HIV transmission, it might be better to inhibit de novo infection of uninfected cells. Since HIV only infects cells bearing the CD4 receptor, CD4 AsiCs (CD4-AsiCs) could, in principle, inhibit infection of all the cells that HIV infects. To test the ability of CD4-AsiCs to inhibit HIV transmission, we engineered AsiCs using 2 high-affinity CD4 aptamers that selectively bind to human, but not mouse, CD4 (33). CD4-AsiCs might inhibit HIV infection in 2 ways: by blocking viral entry via binding to CD4 and by RNAi knockdown of viral genes, host receptors, or other host genes required for viral replication. Here we showed that CD4-AsiCs bearing siRNAs targeting HIV gag and vif or host CCR5 were specifically taken up by $\mathrm{CD} 4^{+}$ cells; knocked down expression of their intended target genes; and inhibited HIV infection in primary $\mathrm{CD}^{+} \mathrm{T}$ cells and macrophages in vitro, in polarized cervicovaginal explants, and in immunodeficient NOD/SCID $\mathrm{Il}_{2 \mathrm{rg}^{-/}}$(NSG) mice reconstituted with human fetal liver $\mathrm{CD} 34^{+}$cells and surgically implanted with human fetal thymic tissue (so-called "BLT mice"; ref. 34). Although the CD4 aptamer on its own inhibited HIV infection to some extent, chimeric RNAs were significantly more effective at preventing transmission to cervicovaginal explants and to BLT mice.

\section{Results}

Synthesis and purification of CD4-AsiCs. CD4-AsiCs were designed with a CD4 aptamer at the $5^{\prime}$ end ligated to the sense (inactive) siRNA strand (Figure 1A and Supplemental Figure 1; supplemental material available online with this article; doi:10.1172/ JCI45876DS1). These were in vitro transcribed (IVT) from a PCR template, using 2 '-fluoropyrimidines to enhance stability in human genital fluid (Supplemental Figure 2) and reduce stimulation of cellular immune sensors that detect foreign nucleic acids. Transcripts were eluted from denaturing SDS-PAGE gels and analyzed by column chromatography and native SDS-PAGE gels
(Supplemental Figure 3), before annealing the antisense (active) siRNA strand. A chimera using an aptamer targeting PSMA (28) was synthesized as a binding control, while scrambled siRNA sequences controlled for gene silencing specificity. CD4-AsiCs engineered with one of the aptamers was consistently more effective than the other (clone 9 vs. clone 12; Supplemental Figure 1, B and C; data not shown; and ref. 33); therefore, unless otherwise indicated, clone 9 was used for subsequent experiments.

CD4-AsiCs are taken up by primary $C D 4^{+}$cells in vitro. To test for siRNA uptake into $\mathrm{CD}^{+}$cells, primary monocyte-derived macrophages (MDMs) and CD4 ${ }^{+} \mathrm{T}$ cells, freshly isolated from the blood of healthy donors, were incubated with CD4-AsiCs labeled with Cy3 at the $3^{\prime}$ terminus of the antisense strand. CD4-AsiCs were efficiently and uniformly taken up by both MDMs (Supplemental Figure 4, A and B) and $\mathrm{CD}^{+} \mathrm{T}$ cells (Figure 1, B and C). PSMAAsiCs were not internalized without transfection (complexation with cationic lipids for MDMs, electroporation for T cells). When added to resting PBMCs, Cy3-labeled CD4-AsiCs were selectively taken up by $\mathrm{CD}^{+}$monocytes and $\mathrm{T}$ cells, but only to a limited extent by $\mathrm{CD}^{+} \mathrm{T}$ cells (Figure 1D). The small subpopulation of circulating $\mathrm{CD}^{+} \mathrm{T}$ cells that took up the Cy3-labeled CD4-AsiCs may represent recently activated $\mathrm{CD}^{+} \mathrm{T}$ cells that express low levels of CD4 (data not shown). We also cannot rule out that staining might be due to cell surface binding without internalization. PSMA-AsiCs on their own were not internalized by any immune cells, except as lipoplexes. In that case, the fluorescent RNA, as expected, was internalized by $\mathrm{CD} 14^{+}$monocytes, but not by $\mathrm{CD}^{+}$ lymphocytes, which are refractory to lipid-based transfection.

CD4-AsiCs knock down target gene expression in primary $\mathrm{CD}^{+}$cells in vitro in a dose-dependent manner. Our next goal was to evaluate CD4AsiC-mediated gene silencing. Primary $\mathrm{CD}^{+}$cells were treated with CD4-AsiCs bearing a CCR5 siRNA, and CCR5 expression was quantified by flow cytometry. CCR5 was knocked down in both MDMs (Supplemental Figure 4, C and D) and CD4 ${ }^{+} \mathrm{T}$ cells (Figure 1, E and F). Gene silencing was specific: CCR5 expression was unchanged when chimeras containing a scrambled siRNA sequence or the PSMA aptamer were tested, or when cells were incubated with the CD4 aptamer alone. As expected, transfection of CCR5 PSMA-AsiCs knocked down CCR5 expression in both cell types. Although silencing in $\mathrm{CD}^{+} \mathrm{T}$ cells was uniform, we observed 2 populations of MDMs: knockdown was virtually complete in one, whereas CCR5 was only partially downmodulated in the other. These 2 populations were also seen with lipid transfection. The reason for this is unclear, but is unlikely to be secondary to differential uptake, since uptake of Cy3-labeled CD4-AsiCs was uniform in macrophages (Supplemental Figure 4B).

Dose-dependent uptake of Cy3-labeled CD4-AsiCs and CCR5 knockdown occurred in both HeLa cells expressing CD4 and CCR5 (data not shown) and primary MDMs (Supplemental Figure 5). Uptake and gene silencing in MDMs after 72 hours were confirmed by fluorescence microscopy, in which Cy3 uptake was found to coincide with CCR5 knockdown, and neither uptake nor gene silencing occurred in MDMs incubated with Cy3-labeled siRNAs on their own (Supplemental Figure 4E). CD4-AsiCs could be readily designed to silence other genes, including the nuclear envelope gene lamin A (Supplemental Figure 6), the pan-leukocyte marker CD45 (Supplemental Figure 7), and the mitotic spindle gene EG5 (data not shown). Dosedependent silencing was restricted to $\mathrm{CD}^{+}$cells, as assessed by immunoblot to measure protein and quantitative RT-PCR 

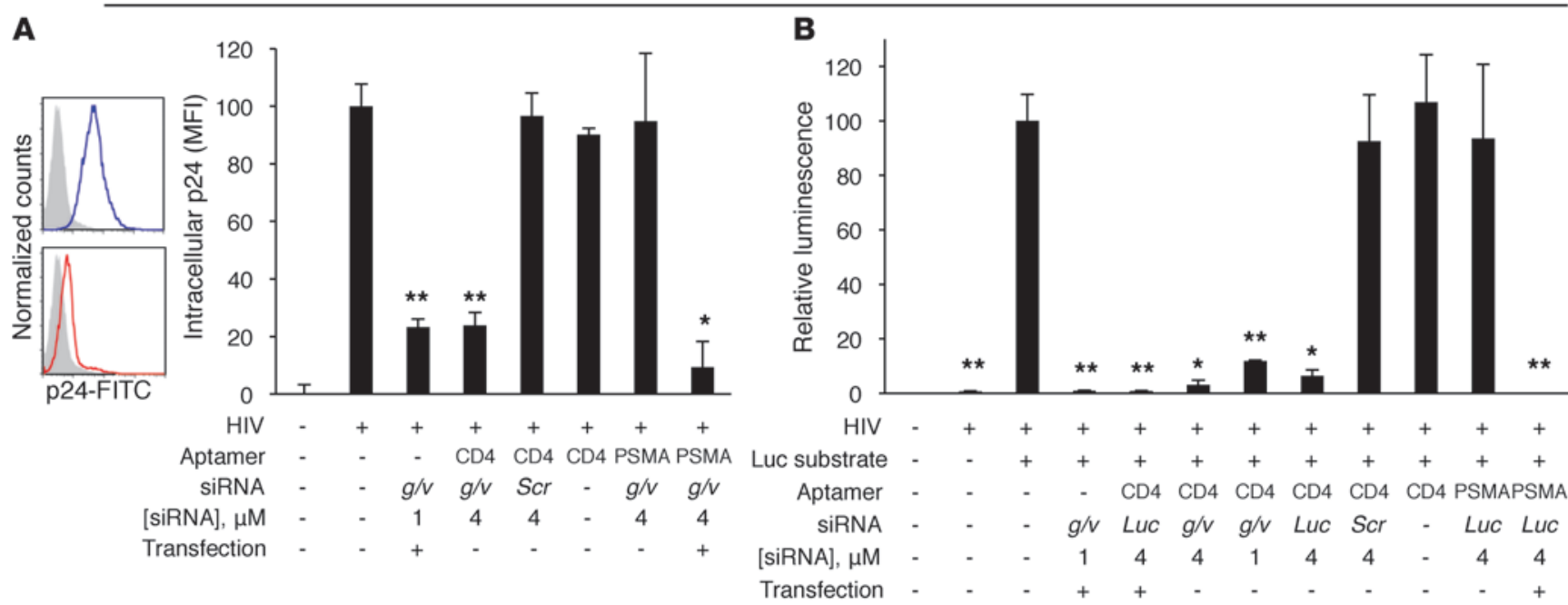

C
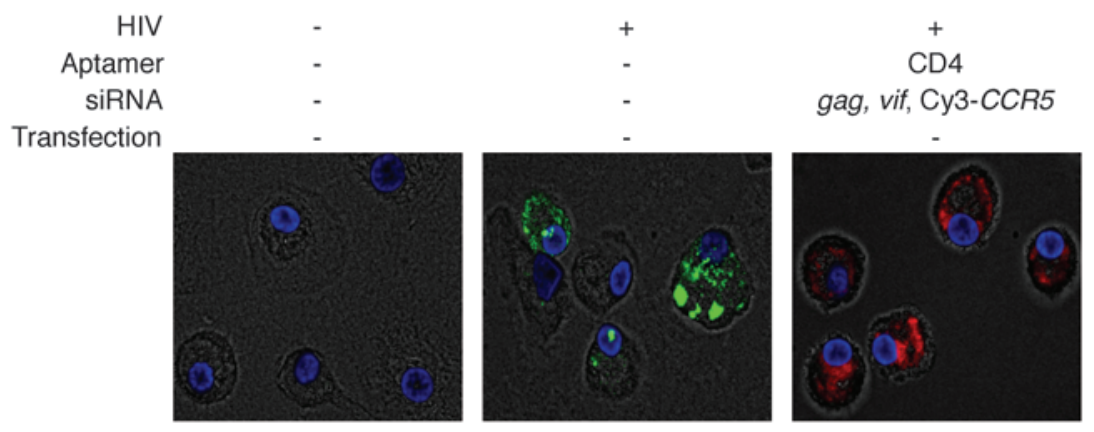

PSMA

Aptamer - $\quad+\quad+-C_{4}$ CD4 CD4 PSMA PSMA

siRNA

[siRNA], $\mu \mathrm{M}$

$g / v \quad g / v \quad S c r \quad-g / v \quad g / v$

(sif

[siRNA], $\mu \mathrm{M}$

Transfection

gag, vif, Суз-CCR5 gag, vif, Суз-CCR5

CD4+ $\mathrm{T}$ lymphocytes

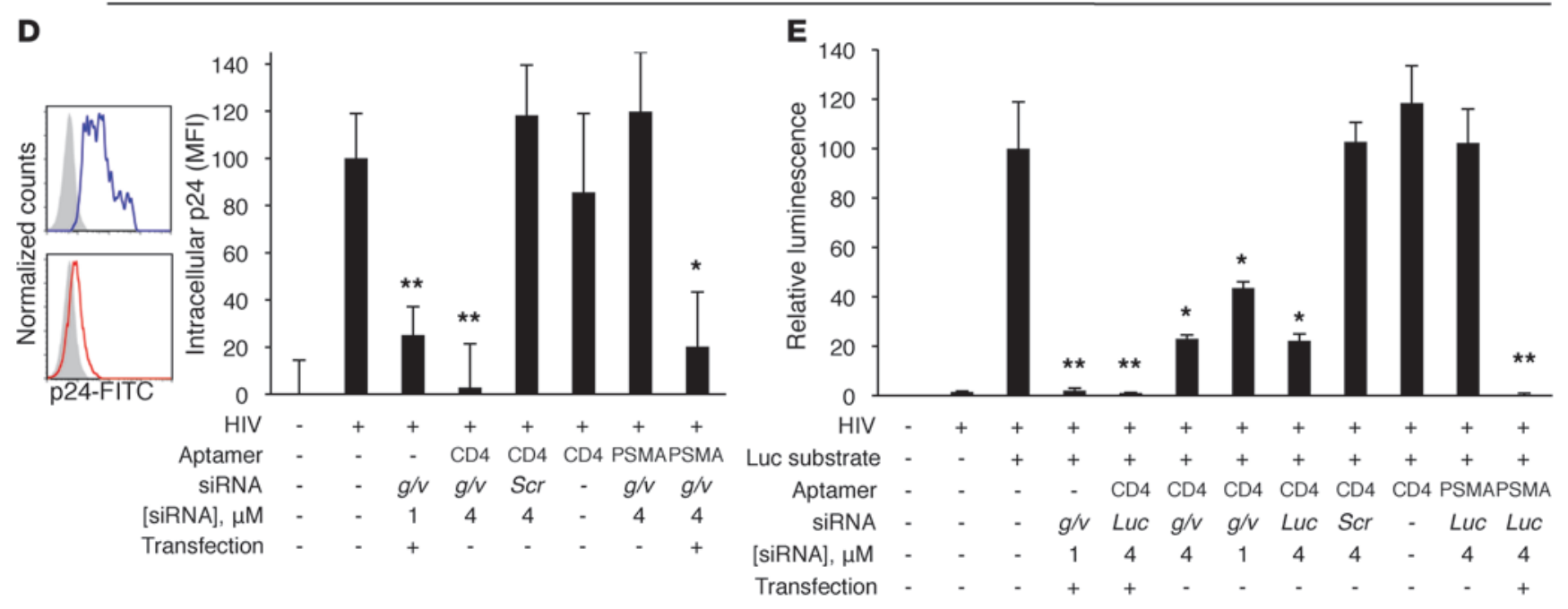




\section{Figure 2}

CD4-AsiCs inhibit HIV replication in vitro. MDMs $(\mathbf{A}-\mathbf{C})$ and $C D 4^{+} \mathrm{T}$ cells (D and E) were infected for 48 hours with HIV-1 $1_{\mathrm{BaL}}$ and HIV-1 $1_{\text {IIIb, }}$, respectively, and then treated with CD4-AsiCs or PSMA-AsiCs bearing gag and vif $(g / v)$ siRNAs. Scrambled siRNA chimeras and CD4 aptamers were controls. Transfection controls used OF (A and $\mathbf{B}$ ) or nucleofection (D and E). (A and D) Intracellular p24 expression, relative to infected mock-treated cultures, was measured by flow cytometry 48 hours later. CD4-AsiCs inhibited HIV replication (mean \pm SEM normalized to mock; $n=3$; ${ }^{\star} P<0.05,{ }^{\star \star} P<0.005$, 2-tailed $t$ test). Insets show representative histograms of MDMs and CD4+ $T$ lymphocytes treated with gag and vif CD4-AsiCs (gray, uninfected; blue, infected mocktreated; red, infected CD4-AsiC-treated). (C) HIV infection was also evaluated by fluorescence in situ hybridization (original magnification, $\times 60$ ) using FITC-labeled probes complementary to HIV genomic RNA. HIV RNA was virtually undetectable in MDMs treated with $4 \mu \mathrm{M}$ total final concentration of CD4-AsiCs. (B and E) To evaluate gene silencing independently of the effect of the CD4 aptamer on blocking viral entry, HIV replication was assessed by infection with VSV(G)-pseudotyped virus containing a luciferase reporter gene. Primary MDMs and $\mathrm{CD}^{+} \mathrm{T}$ cells were pretreated for 48 hours before infection with $4-\mu \mathrm{M}$ mixtures of CD4- or PSMA-AsiCs targeting gag and vif or containing scrambled siRNAs. Luciferase activity, measured 48 hours later, was significantly inhibited in cells treated with CD4-AsiCs directed against either viral or luciferase genes (mean \pm SEM normalized to mock; $\left.n=3 ;{ }^{*} P<0.05,{ }^{* \star} P<0.005\right)$.

(qRT-PCR) for mRNA. Based on these results, we expect that CD4-AsiCs could be used to manipulate expression of virtually any gene in human $\mathrm{CD}^{+}$immune cells.

CD4-AsiCs are Dicer substrates and are processed intracellularly into functional siRNAs that use the RNAi pathway to direct $m R N A$ cleavage. To understand the mechanism of CD4-AsiC-mediated silencing, we first tested whether these chimeras are substrates for the endoribonuclease Dicer, which processes longer endogenous RNA precursors to short 20- to 25-nt RNAs as part of the RNAi pathway in the cell. When CD4-AsiCs bearing CCR5 siRNAs were incubated with recombinant Dicer, they were virtually completely digested to an expected approximately 21 - to 23 -nt cleavage product that migrated like a CCR5 siRNA (Supplemental Figure 8A). Treatment of primary $\mathrm{CD}^{+} \mathrm{T}$ cells with ${ }^{32} \mathrm{P}$-end-labeled CD4-AsiCs also resulted in their processing to an approximately 21- to 23-nt duplex RNA (Supplemental Figure 8B), which suggests that similar Dicer cleavage also occurs within cells. Gene silencing by CD4-AsiCs also depended on intracellular Dicer expression in HCT-116 cells, since knockdown of lamin A by CD4-AsiCs only occurred in WT cells, but not in Dicer $^{-1}$ cells (Supplemental Figure 8C and ref. 35). To confirm that AsiC-mediated silencing was caused by siRNA-directed cleavage of target gene mRNA, modified $5^{\prime}$-rapid amplification of cDNA ends (5'-RACE; ref. 36) was used to analyze RNA isolated from primary MDMs treated with CCR5 CD4-AsiCs for CCR5 mRNA cleavage fragments (Supplemental Figure 9). An amplified CCR5 RNA fragment of the expected size was detected 72 hours after adding the CCR5 CD4-AsiCs. Sequencing of the amplified fragments confirmed that cleavage occurred $10 \mathrm{nt}$ from the $5^{\prime}$ end where the CCR5 antisense strand bound, at the expected site (37). Thus, we conclude that CD4-AsiCs are processed by Dicer to release functional siRNA duplexes that direct target mRNA cleavage via the RNAi pathway.

$\mathrm{CD} 4-A$ siCs inhibit HIV replication in primary $\mathrm{CD}^{+}$cells in vitro. Inhibiting HIV infection would be a stringent test of effective gene knockdown in $\mathrm{CD}^{+}$cells. We first tested whether treatment with a mixture of CD4-AsiCs against HIV gag and vif (Figure 2) or against
CCR5 (Supplemental Figure 10) could suppress viral replication in an established HIV infection. Primary cells were infected with HIV-1 48 hours before CD4-AsiC treatment. Viral production was assessed by flow cytometry analysis of intracellular p24 capsid Ag (p24-Ag) or by p24 Ag ELISA of the culture supernatant. CD4-AsiCs against viral genes inhibited HIV replication in $\mathrm{MDMs}$ and $\mathrm{CD} 4^{+} \mathrm{T}$ cells (Figure 2, A and D). siRNAs against CCR5 also inhibited HIV infection in primary MDMs (Supplemental Figure 10), and siRNAs against viral genes inhibited infection in HeLa-CD4 and Jurkat cells in a dose-dependent manner (Supplemental Figure 11). There was no antiviral effect using chimeras containing PSMA aptamers or scrambled siRNAs. Viral inhibition and RNA internalization were confirmed by FISH for HIV RNA and Cy3 fluorescence, respectively, in MDMs treated with a cocktail of gag, vif, and Cy3-labeled CCR5 CD4-AsiCs, but not with a cocktail of PSMA-AsiCs (Figure 2C).

These CD4 aptamers bind CD4 principally via its V4 domain, but also partially block the receptor's V1 gp120-binding domain $(33,38)$. Therefore, CD4-AsiCs could inhibit HIV infection by 2 mechanisms: by blocking HIV entry via inhibition of HIV gp120 binding to CD4, or by knocking down either viral genes or host genes required for viral entry or replication. In fact, preinfection treatment of cells with CD4-AsiCs bearing scrambled siRNAs or with CD4 aptamers lacking an siRNA tail partially inhibited infection in HeLa-CD4 cells (data not shown), which suggests that blocking HIV binding to CD4 by the aptamer contributes to overall inhibition of HIV infection. To evaluate the gene silencing component of chimera-mediated HIV suppression on its own, we pretreated cells with AsiCs before challenging with a CD4-independent single-round VSV(G)-pseudotyped HIV-1 virus encoding a luciferase reporter gene. In both MDMs and $\mathrm{CD}^{+} \mathrm{T}$ cells, CD4-AsiCs bearing gag and vif or luciferase siRNAs significantly decreased luciferase activity compared with all control conditions (Figure 2, B and E). Therefore, the CD4-AsiCs encoding either viral or CCR 5 siRNAs likely inhibit HIV infection by both blocking entry and gene knockdown.

CD4-AsiCs do not alter expression of lymphocyte activation markers. CD4-AsiCs might be a useful tool for genetic manipulation of hard-to-transfect $\mathrm{CD} 4^{+}$cells in order to study the effect of knocking down one gene at a time. Ideally, treatment with CD4-AsiCs should not alter CD4 surface expression or cause lymphocyte activation, which would confound interpretation of gene silencing experiments. Activation of $\mathrm{CD}^{+} \mathrm{T}$ cells by CD4-AsiCs would also make them undesirable for preventing or treating HIV infection, since activated $T$ cells are more susceptible to infection. Since CD4-AsiCs contain a single receptor binding site and T cell activation requires receptor cross-linking, we would not expect CD4AsiC treatment to activate the cells. To evaluate this, uninfected $\mathrm{CD}^{+} \mathrm{T}$ cells treated with CD4-AsiCs directed against exogenous viral genes were assayed by flow cytometry over 2 days for changes in surface expression of CD4 and other markers of lymphocyte activation. Neither CD4 nor other cell surface receptors (CD3, CD45, CD25, and CD69) were significantly changed compared with mock-treated controls (Supplemental Figure 12).

CD4-AsiCs inhibit HIV replication in polarized cervicovaginal explants. To assess whether CD4-AsiCs can penetrate the vaginal epithelium and specifically knock down gene expression in $\mathrm{CD}^{+}$cells in intact human tissue, we treated the apical surface of polarized agaroseembedded human cervicovaginal explants, obtained from normal hysterectomy specimens $(39,40)$, with Cy3-labeled CCR5 CD4-AsiCs twice in a 24-hour interval. Cy3 fluorescence and CCR5 expression were measured in isolated $\mathrm{CD}^{+}$and $\mathrm{CD} 8^{+} \mathrm{T}$ cells and in $\mathrm{CD} 14^{+}$ 
A

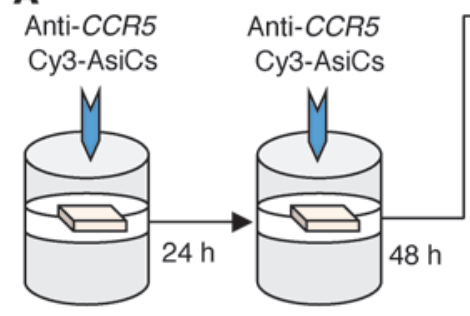

B $\mathrm{CD}^{+} / \mathrm{CD} 14^{+}$

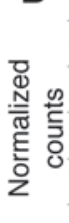

Flow cytometry of $\mathrm{CD}^{+}$cells
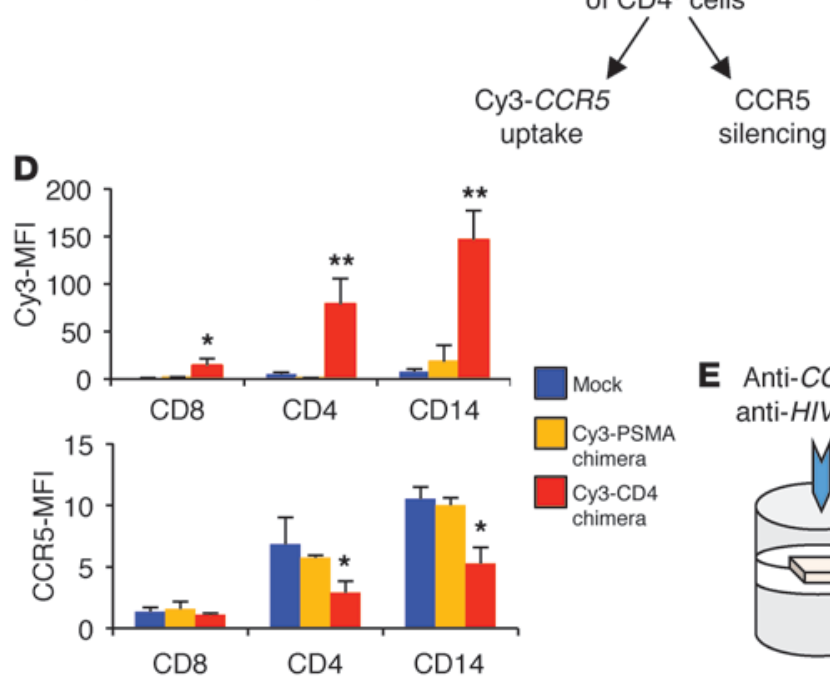

E Anti-CCR5 or anti-HIV AsiCs

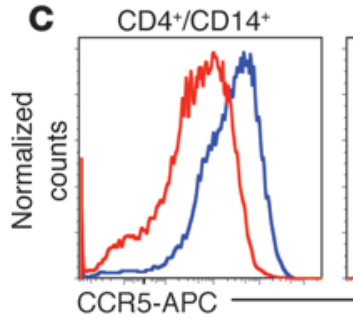

$\mathrm{CD}^{+} / \mathrm{CD}^{+}$

$\mathrm{CD}^{+} / \mathrm{CD}^{+}$

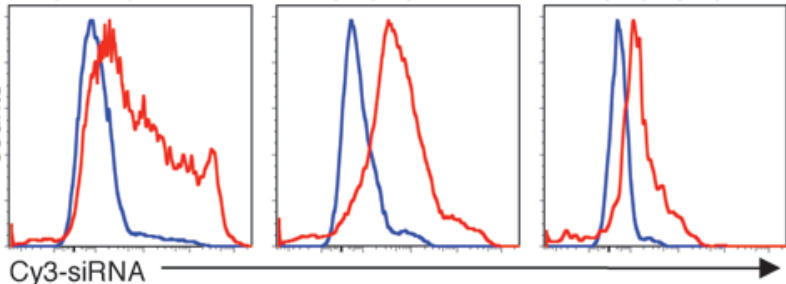

C $\mathrm{CD}^{+} / \mathrm{CD} 14^{+} \quad \mathrm{CD}^{+} / \mathrm{CD}^{+}$

$\mathrm{CD}^{+} / \mathrm{CD}^{+}$
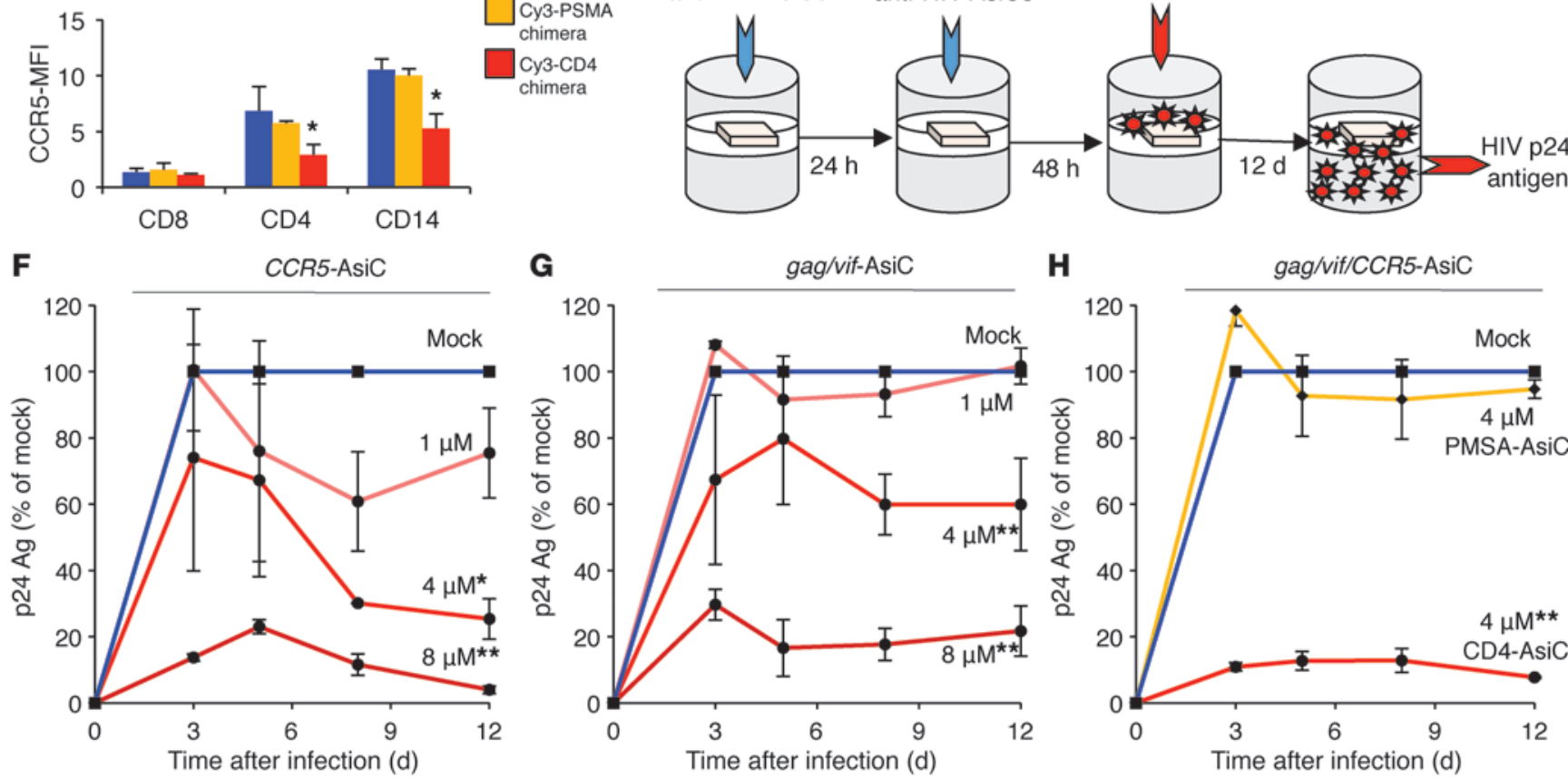

G

gag/vif-AsiC
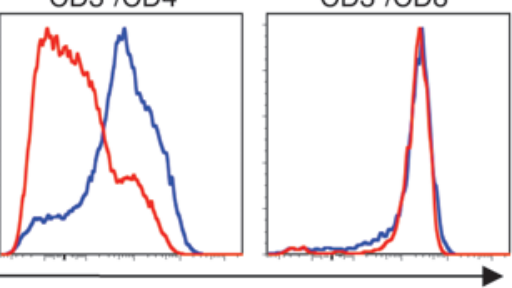

Суз-CD4

chimera

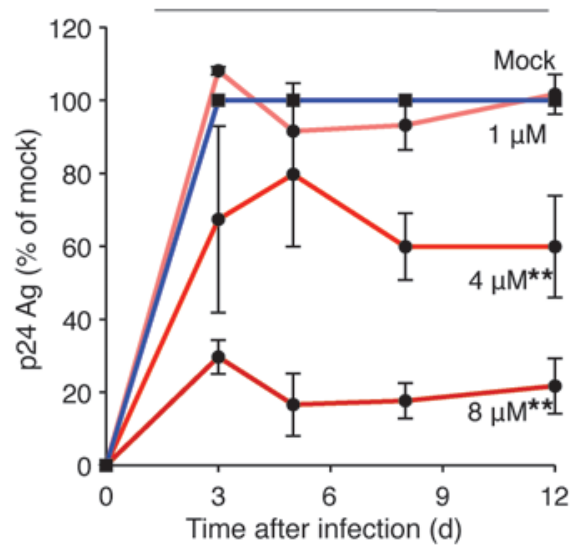

J
H

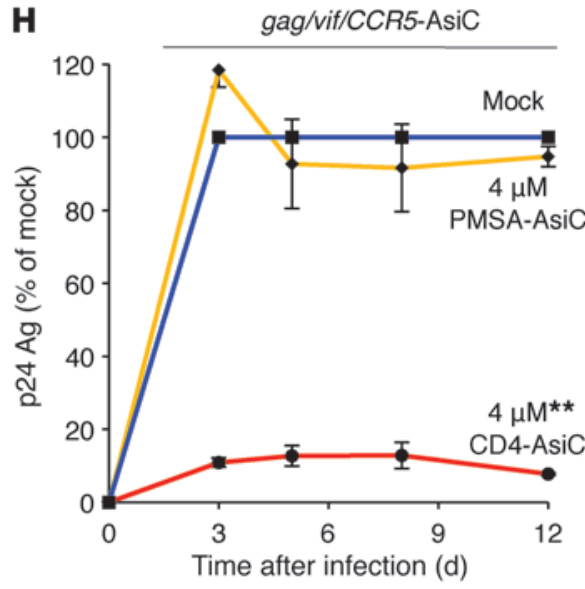

Day 5 after treatment
I

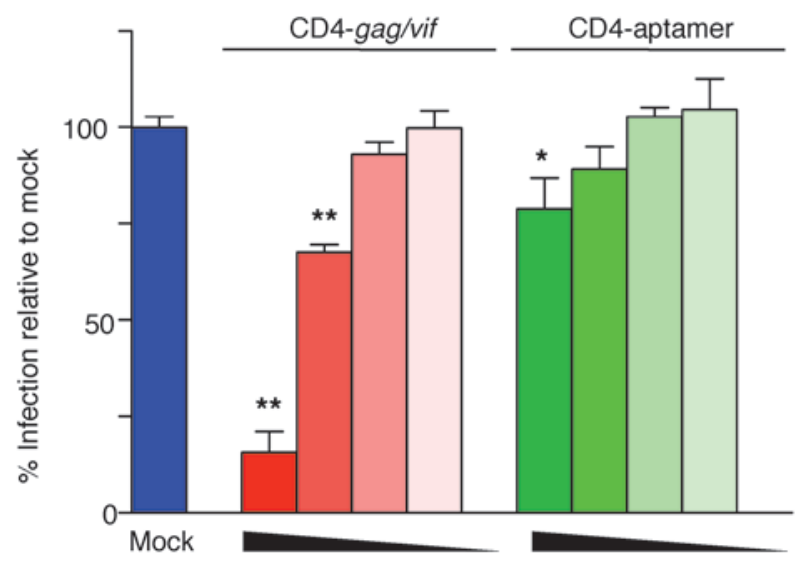

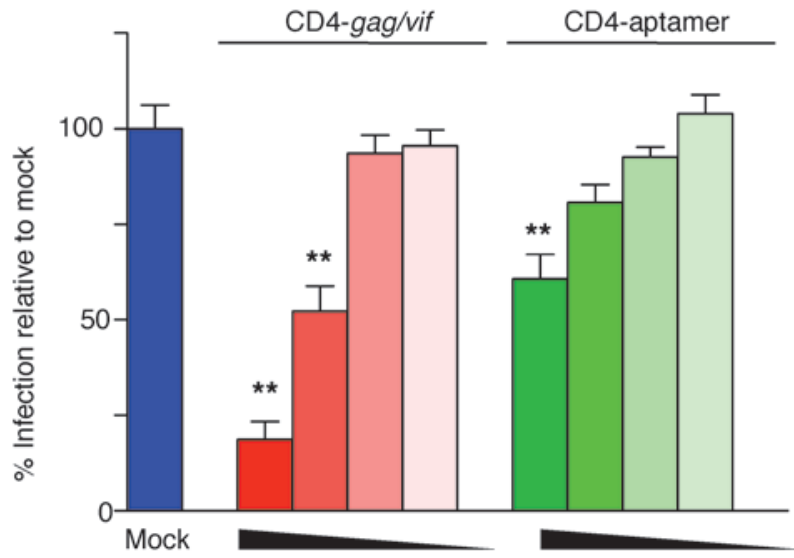




\section{Figure 3}

CD4-AsiCs inhibit HIV replication in human cervicovaginal explants. (A-D) Cy3-labeled CD4-AsiCs or PBS was applied to the epithelium of agarose-embedded polarized explants 72 and 48 hours before flow cytometry of single-cell suspensions analyzed for Сy3 uptake (B) and CCR5 knockdown (C). Uptake was highest in $\mathrm{CD}^{+}{ }^{+} \mathrm{CD} 4^{+}$ $T$ cells and $\mathrm{CD} 4{ }^{+} \mathrm{CD} 14^{+}$macrophages, and CCR 5 knockdown occurred only in CD4+ populations. (D) MFI (mean \pm SEM normalized to mock; ${ }^{\star} P<0.05,{ }^{* \star} P<0.01,2$-tailed $t$ test). Data from quadruplicate samples from 1 donor are representative of results from 3 donors. (E-H) HIV challenge experiment. Polarized vaginal explants from 3 donors were pretreated with indicated total concentrations of CD4-AsiCs targeting CCR5 (F), gag and vif $(\mathbf{G})$, or all 3 genes $(\mathbf{H})$ for 48 hours before HIV-1 $1_{\mathrm{BaL}}$ challenge. Viral replication, measured by p24 Ag ELISA of the lower transwell culture medium, showed specific dose-dependent inhibition by CD4-AsiCs against CCR5 (F) or gag and vif (G) compared with mocktreated controls. At the same total concentration, AsiCs against CCR5 and gag and vif $(\mathbf{H})$ was more effective than either CCR5 or viral CD4AsiCs alone. PSMA-AsiCs did not inhibit viral replication. Shown are p24 Ag levels (mean \pm SEM normalized to mock; ${ }^{\star} P<0.05,{ }^{* *} P<0.01$, 2-tailed $t$ test). (I and $\mathbf{J}$ ) The CD4 aptamer was compared with the gag and vif chimera by treating vaginal explants with serial 2-fold dilutions $(1-8 \mu \mathrm{M})$ of each. p24 Ag ELISA was measured and normalized as above. Data are representative of independent experiments from 2 donors, performed in quadruplicate $\left({ }^{\star} P<0.05\right.$, ${ }^{* \star} P<0.0001$, 1 -way ANOVA with Dunnett multiple-comparison test).

macrophages by flow cytometry 2 days after the second treatment (Figure 3, A-D). Cy3-labeled CD4-AsiCs were specifically taken up by $\mathrm{CD}^{+} \mathrm{T}$ cells and macrophages in situ and uniformly knocked down CCR5 expression. Despite nominal uptake by $\mathrm{CD}^{+} \mathrm{T}$ cells, no gene silencing was observed in this population. Neither uptake nor CCR5 silencing was seen in tissues treated with PSMA-AsiCs (Figure 3D). Topical application of CD4-AsiCs encoding a CD45 siRNA to explants also specifically knocked down CD45 expression only in $\mathrm{CD}^{+} \mathrm{T}$ cells and macrophages (data not shown).

To determine whether antiviral CD4-AsiCs can block HIV transmission in intact vaginal tissue, the epithelial surface of polarized explants was treated twice with CD4-AsiCs containing siRNAs targeting CCR5, gag and vif, or all 3 genes before challenge with $\mathrm{HIV}_{\mathrm{BaL}}$ 48 hours later. Mucosal viral replication, as assessed by p24 Ag ELISA of the explant culture medium, was inhibited by CD4-AsiC treatment in a dose-dependent manner (Figure 3, F-H). The triple cocktail administered at the same total concentration was more effective than the CCR5 or the antiviral CD4-AsiCs alone. A triple cocktail of PSMA-AsiCs did not inhibit HIV transmission to the tissue. To assess the antiviral contribution of the CD4 aptamer, we compared viral inhibition by the CD4 aptamer and the CD4AsiCs encoding gag and vif siRNAs in a dose response study. The CD4 aptamer on its own inhibited HIV transmission in situ, but was about 2- to 4-fold less effective than the CD4-AsiCs (Figure 3, I and J), which suggests that although blocking CD4 binding contributes to HIV inhibition, the major antiviral activity of the CD4AsiC is due to gene knockdown.

CD4-AsiCs do not trigger an innate immune response in buman cervicovaginal tissue. Depending on their sequence, concentration, chemical modifications, and delivery vehicle, siRNAs can trigger innate immune sensors that recognize foreign nucleic acids or cause cytotoxicity and tissue damage (41). Moreover, the demonstrated antiviral effect could be a side effect of IFN induction by TLR or RIG-I activation. To examine whether CD4-AsiCs have any adverse or unanticipated off-target effects, we treated polarized human explants with gag and vif CD4-AsiCs at the same concentration that inhibited HIV inhibition $(4 \mu \mathrm{M})$ and evaluated tissue injury and inflammation by hematoxylin and eosin staining, cytotoxicity by LDH release, and induction of innate immune response genes (Supplemental Figure 13). The explants were treated with poly(I:C) as a positive control and mock treated as a negative control. There was no evidence of tissue injury or inflammation or cytotoxicity. Moreover, CD4-AsiCs did not induce IFNB or IFNG, inflammatory cytokines (IL6,IL8, and IL12), or IFN-responsive genes (IP-10, OAS1, and STAT1), as measured by sensitive qRT-PCR of RNA extracted from whole tissue harvested at their expected peaks, 6 and 24 hours after treatment. Contrary to a previously published report (28), PSMA-AsiCs stimulated IFNB expression at 6 hours and $I P-10$ at 24 hours, likely reflecting the higher sensitivity of qRT-PCR relative to ELISA for measuring cytokine induction.

CD4-AsiCs suppress target gene expression in $\mathrm{CD}^{+}$cells in vivo. To validate the antiviral properties of CD4-AsiCs in vivo, we used the BLT humanized mouse model (42). A mixture of CD4-AsiCs bearing CD45 siRNAs and Cy3-labeled CCR5 siRNAs were applied IVAG to NOD/SCID-BLT mice, 72 hours and 48 hours prior to sacrifice, each at doses ranging from 5 to $80 \mathrm{pmol}$ (Figure 4A). To assess cell-specific siRNA delivery and gene knockdown in vivo, Cy3 fluorescence and CD45 and CCR5 expression in subpopulations of $\mathrm{CD}^{+}$and $\mathrm{CD}^{+}$cells were evaluated by flow cytometry of singlecell suspensions of vaginal tissue and compared with mock-treated control mice (Figure 4, B and C). Cy3 fluorescence uptake was uniform in $\mathrm{CD}^{+} \mathrm{CD}^{+} \mathrm{T}$ cells and $\mathrm{CD} 14^{+} \mathrm{CD} 4^{+}$tissue macrophages, but was absent in tissue $\mathrm{CD} 3^{+} \mathrm{CD} 8{ }^{+} \mathrm{T}$ cells. Dose-dependent CCR5 and CD45 knockdown was observed in $\mathrm{CD}^{+} \mathrm{T}$ cells, but not in $\mathrm{CD}^{+} \mathrm{T}$ cells. At the highest CD4-AsiC dose, cell surface CD45 and CCR5 MFI in CD4 ${ }^{+} \mathrm{T}$ cells was reduced 12 - and 5-fold, respectively, compared with mock-treated controls.

Topically applied CD4-AsiCs inhibit vaginal transmission of HIV to bumanized mice. To test whether topically applied CD4-AsiCs protect humanized mice from vaginal HIV challenge, 4 NSG-BLT mice were treated with a combination of CD4-AsiCs targeting CCR5, gag, and vif prior to IVAG challenge with $10^{5}-\mathrm{TCID}_{50} \mathrm{HIV}_{\text {JR-CSF }}$ (Figure 4D), a viral dose that uniformly infects control mice (data not shown and refs. 43, 44). The CCR5 AsiCs were administered 48 and 24 hours prior to challenge, and the viral gene-targeting AsiCs were administered 24 hours before and 4 hours after IVAG challenge. We treated 4 mice with equivalent doses of CD4 aptamers lacking siRNA conjugates and 4 mice with PBS using the same dosing regimen. In the 12 weeks of observation after challenge, all the aptamer- and AsiC-treated mice survived, while 2 of the mock-treated mice died (Figure 4E). Whereas all the control and aptamer-treated mice became infected and had detectable HIV p24 antigenemia 2-4 weeks after infection, none of the CD4-AsiC-treated mice developed detectable p24 antigenemia (Figure 4F). Plasma viral burden, assessed by sensitive qRT-PCR assay for HIV gag mRNA, was detected at very low levels and only episodically several months later in the 2 of 4 CD4-AsiC-treated mice that became infected, a significant reduction compared with the mock-treated or aptamer-treated animals (Figure 4G). All the control mice and 2 of the 4 aptamertreated mice showed dramatic depletion of circulating $\mathrm{CD}^{+} \mathrm{T}$ cells, whereas all mice treated with CD4-AsiCs and the other 2 aptamer-treated mice maintained relatively normal CD4 counts and stable CD4/CD8 ratios (Figure 4H and Supplemental Figure 14). Although we did not directly determine the cause of death of the mock-treated mice, the profound depletion of their circulating 
A NOD/SCID-BLT NOD/SCID-BLT
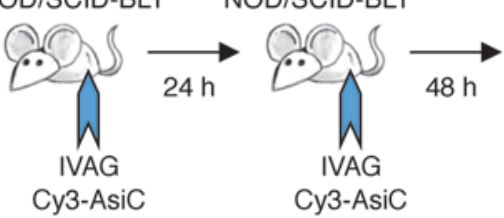

Collagenase digest

of vaginal tissue

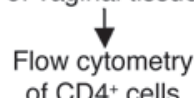

of $\mathrm{CD}^{+}$cells
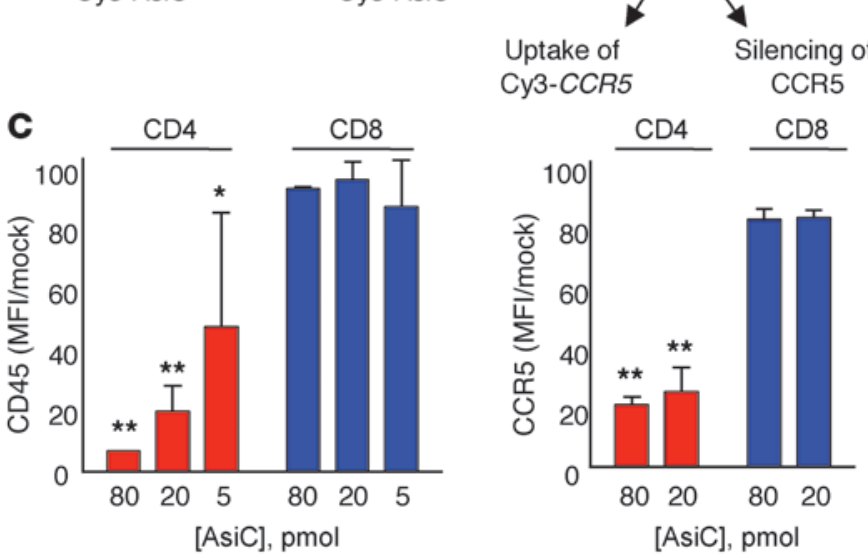

D

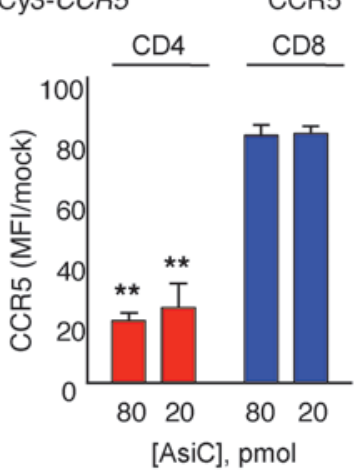

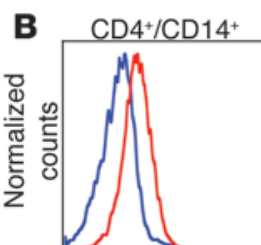
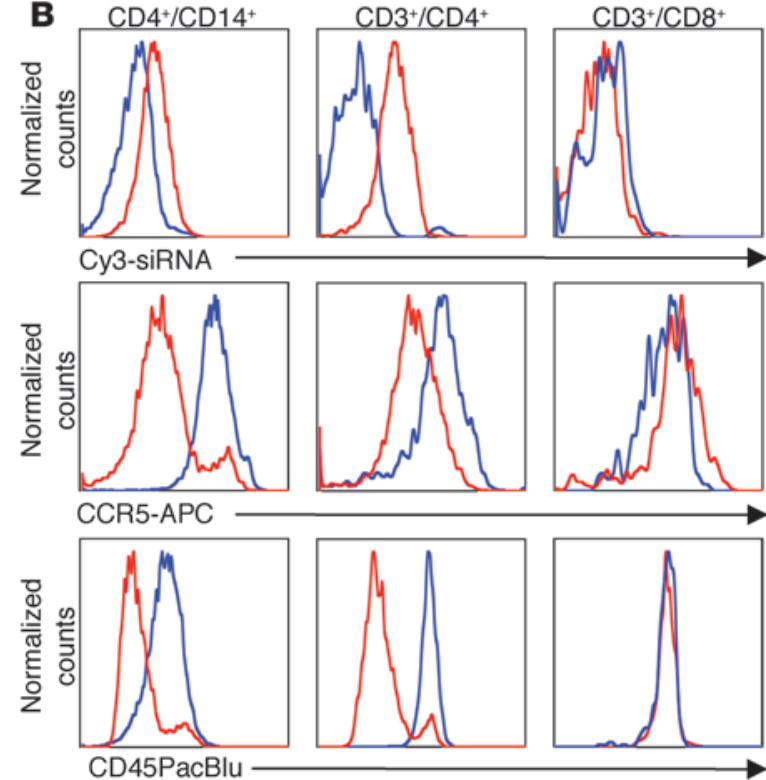

E

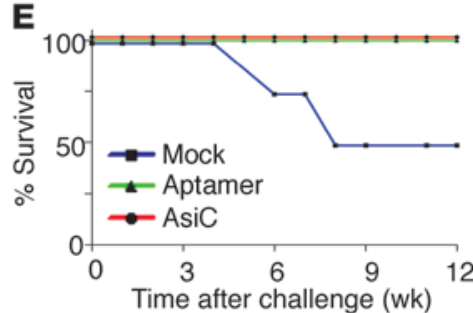

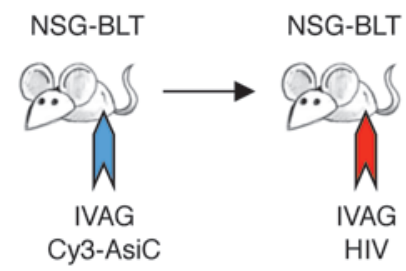

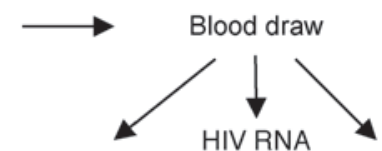

Plasma p24 T cell counts

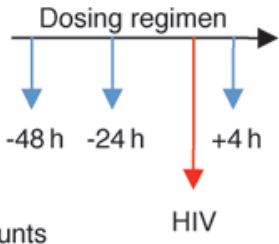

Asic
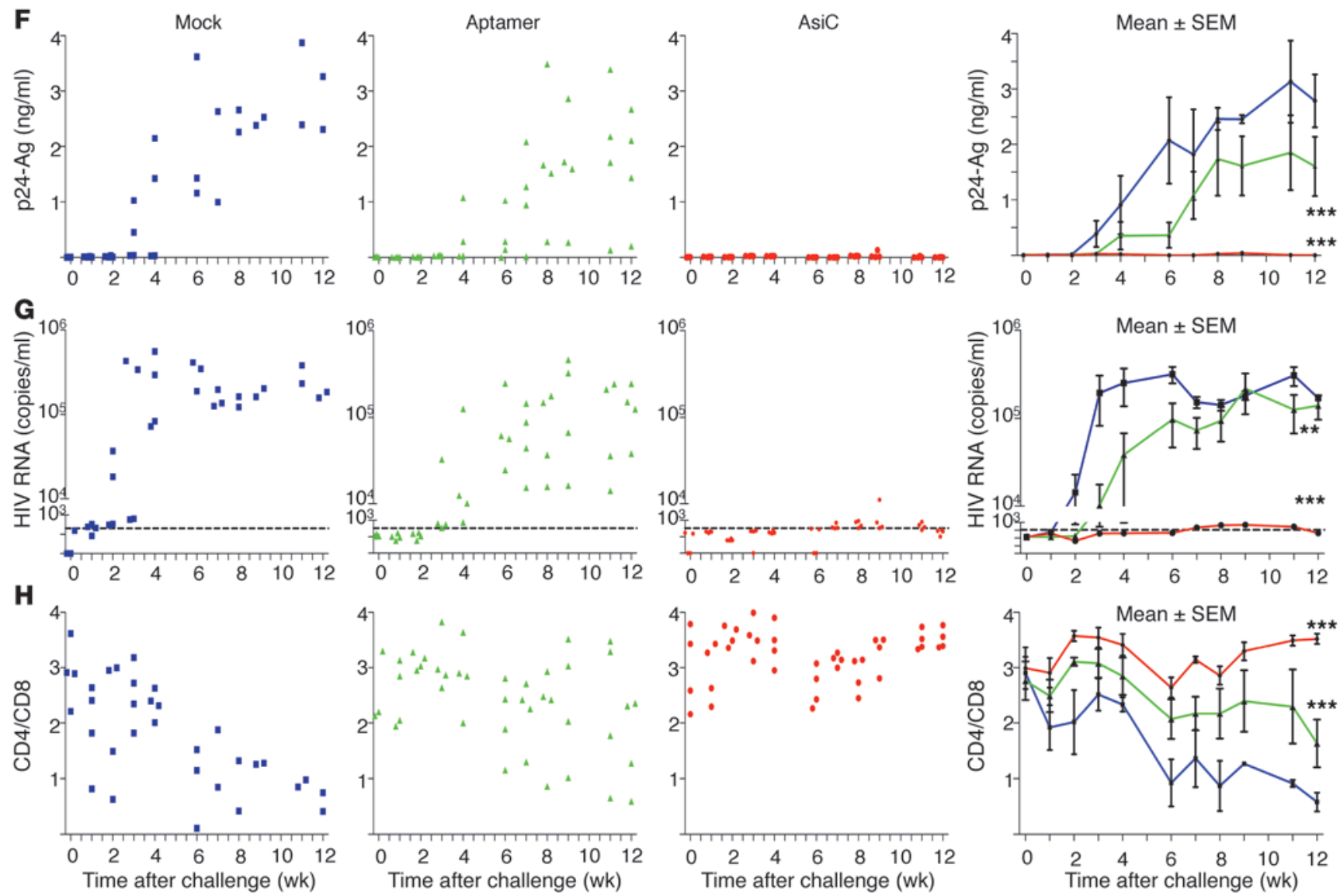


\section{Figure 4}

CD4-AsiCs applied IVAG inhibit vaginal HIV transmission to humanized BLT mice. (A-C) NOD/SCID-BLT mice (2 per group) were treated twice IVAG with the indicated $5-80$ pmol mixture of CD4 chimeras bearing Cy3-labeled CCR5 siRNA and CD45 siRNA. 2 days later, a single-cell suspension extracted from vaginal tissue was analyzed by flow cytometry for Cy3-labeled siRNA uptake and target gene knockdown. (B) Representative histograms (blue, mock; red, 80-pmol dose). (C) MFI of CD4+ $\mathrm{T}$ cells in the vaginal tissue of AsiC-treated mice showed a dose-dependent decrease of both CD45 and CCR5 expression relative to mock-treated controls, but the MFI of CD8+ $\mathrm{T}$ cells did not change (mean \pm SEM; ${ }^{*} P<0.01$, ${ }^{\star \star} P<0.001$, 1 -way ANOVA with Dunnett multiple-comparison test). (D) HIV challenge experiment. NSG-BLT mice were treated IVAG with CCR5 CD4-AsiCs 48 hours ( $80 \mathrm{pmol})$ and 24 hours (40 pmol) before, and 40 pmol each of $g a g$ and vif chimeras 24 hours before and 4 hours after, IVAG challenge with $\mathrm{HIV}_{\mathrm{JR}-\mathrm{CSF}}$. CD4-AsiC treatment was compared with treatment with the same concentration of CD4 aptamer or mock treatment with PBS ( $n=4$ per group). Mice were observed for 12 weeks after HIV challenge. Shown is the effect of treatment on (E) survival; (F) plasma HIV $\mathrm{Ag}$, assessed by ELISA; (G) plasma HIV RNA, analyzed by qRT-PCR (dashed line denotes limit of detection); and (H) circulating CD4 ${ }^{+} / \mathrm{CD}^{+}$ T cell ratio. Data are mean \pm SEM. ${ }^{* *} P<0.001,{ }^{* * *} P<0.0001$ vs. mock, 2-way ANOVA with Bonferroni correction.

$\mathrm{CD}^{+} \mathrm{T}$ cells in the 2 weeks prior to death may have been responsible. Thus, topical application of a mixture of CD4-AsiCs targeting CCR 5 and HIV genes provided protection against vaginal challenge. Although the detection of plasma viremia and development of $\mathrm{CD}^{+} \mathrm{T}$ cell depletion were delayed in the aptamer-treated mice, their viral load was not significantly less than in mock-treated mice by the end of the observation period. Therefore, the CD4 aptamer, which inhibits HIV from binding to CD4, was substantially less effective than the chimera, which can both block binding and suppress entry and viral replication by gene knockdown.

\section{Discussion}

Delivery remains a significant obstacle to the clinical development of siRNA-based drugs. Although cholesterol-conjugated siRNAs silence gene expression without apparent toxicity in mucosal epithelial cells and can be used to prevent HSV-2 transmission (24), this approach would not inhibit HIV transmission, since cholesterol-conjugated siRNAs do not transfect the cells that HIV infects. siRNAs can be delivered into immune cells by receptor-mediated endocytosis, by either complexing siRNAs to antibody fusion proteins or encapsulating siRNAs into liposomes or other nanoparticles bearing targeting antibodies or ligands to cell surface receptors $(25-27,45)$. Here we demonstrated that an alternate approach chimeric RNAs composed of an aptamer linked to an siRNA delivered siRNAs and knockdown target gene expression specifically in primary $\mathrm{CD} 4^{+} \mathrm{T}$ cells and macrophages, regardless of activation state, in vitro, in intact human explants and in humanized mice. Importantly, the CD4-AsiCs were able to inhibit vaginal HIV transmission to humanized mice. Although only 2 of 4 CD4AsiC-treated mice maintained undetectable virus throughout the 12-week period, the transient viremia, detected only after 7-9 weeks after infection, was just above the limit of detection. All CD4-AsiCtreated mice showed preserved $\mathrm{T}$ cell counts, and none had measurable antigenia at any time. These promising results were achieved without any optimization of the CD4-AsiCs for CD4 binding or gene silencing sequences, using an extremely high challenge virus dose that gave uniform infection of control mice. It may be easier to prevent sexual HIV transmission in humans, which is very inefficient, requiring hundreds of exposures for each transmission event, and where usually only a single virion is able to establish a foothold in the host (46). Protection was achieved here with a highest dose of approximately $0.2 \mathrm{mg} / \mathrm{kg}(120 \mathrm{pmol})$, which is a feasible dose for small RNA drugs, but which might be reduced even further by drug optimization. Future studies in humanized mice will evaluate how long gene silencing in the genital tissue and protection lasts in order to determine whether RNAi-based microbicides could be intermittently dosed with acceptable compliance.

Targeted delivery has the potential dual advantage of reduced toxicity to bystander cells and reduced effective dose. What delivery approach is preferable for clinical or research purposes is difficult to determine $\mathrm{ab}$ initio and may depend on the application. Chimeric RNAs have the advantage of being a single molecule rather than a complex mixture, are less likely to be immunogenic than proteins, and are more straightforward to purify and less costly to produce than RNAs that need to be formulated with proteins, nanoparticles, or liposomes. CD4-AsiCs were shown to knock down 2 viral genes, 1 transgene (luciferase), and 4 host genes (CCR5, $C D 45$, lamin $A$, and EG5) and can likely be designed to inhibit the expression of any gene. The kinetics of target gene suppression may differ between targets, depending on target gene mRNA and protein stability. For example, CCR5 and CD45 surface protein expression was not appreciably reduced until 72 hours after CD4AsiC treatment. However, mRNA levels of these genes, when measured by qRT-PCR, declined within a day.

This study builds on previous studies that used intravenous injection of PSMA-AsiCs to transfect human prostate cancer cells in an orthotopic mouse tumor model $(28,29)$ or that used HIV gp120-AsiCs to transfect HIV-infected CD4 $4^{+}$cells in vitro and in vivo (30-32). Based on these studies, the CD4-AsiCs developed here likely could be used for systemic gene silencing in circulating immune cells. If CD4-AsiCs perform as well as we expect, they may be a potent tool for genetic manipulation (which has been so powerful for understanding mouse immunology by use of knockout mice) to study the role of individual molecules in complex human immune responses in humanized mice in vivo. Because CD4-AsiCs do not appear to perturb CD 4 cell surface expression or alter other immune receptors that are sensitive indicators of immune activation, it should be possible to test the effect of knocking down one gene product at a time. The lack of cellular toxicity of CD4-AsiCs might also make them an attractive alternative to electroporation for in vitro transfection of $\mathrm{CD}^{+}{ }^{+} \mathrm{T}$ cells. The clone 9 aptamer also recognizes rhesus macaque $\mathrm{CD} 4$, and the corresponding CD4AsiC knocks down gene expression in rhesus PBMCs in vitro (L.A. Wheeler, unpublished observations), which suggests that CD4-AsiCs could also be used to study SIV or SHIV infection and immune responses in nonhuman primates.

The micromolar concentrations of CD4-AsiCs used here for in vitro gene knockdown and HIV infection inhibition studies are somewhat higher (about 2-10 times more) than those published in some previous reports $(3,24,28,31)$. The higher concentration could be due to differences in target cell type. The CD4-AsiCs have not been optimized, and improvements in CD4-AsiC design or synthesis could increase silencing efficiency. Modifications might include optimizing the aptamer or siRNA sequence, altering the linker joining the aptamer to the siRNA, interchanging the 2 siRNA strands, or replacing the double-stranded siRNA with a stem-loop that mimics endogenous miRNA structures. Some of these changes 
have been used in earlier studies, where the optimal AsiC construct depended on the particular siRNA sequence $(29,31)$. Studies are in progress to understand how CD4-AsiCs are taken up and delivered to the cytosol, where they interact with the endogenous RNAi machinery. To optimize CD4-AsiC design, it will be helpful to compare not only the efficiency of gene silencing, but also the efficiency of intracellular processing to the mature siRNA and its incorporation into the RNA-induced silencing complex. It may also be helpful to shorten the construct to make chemical synthesis more practical. We plan to test whether any part of the aptamer sequence can be deleted without losing binding affinity. Another approach would be to synthesize the aptamer and siRNA separately, using complementary adapter sequences to join them $(30,31)$.

Despite the high concentrations required in vitro, CD4-AsiCmediated in vivo silencing and protection from HIV infection required significantly lower doses (about 7-25 times less) than were used to inhibit HSV-2 transmission with lipoplexed or cholesterol-conjugated siRNAs $(23,24)$. The stability of the CD4AsiCs over 36 hours in human vaginal fluid suggests that further stabilization for topical use may not be required. However, the in vivo half-life in the blood and other body fluids and within cells, as well as the efficiency and durability of gene knockdown, might be improved by further chemical modifications, such as introduction of $2^{\prime}-\mathrm{OCH}_{3}$ to purines on the active strand. For systemic use, chemical conjugation to cholesterol or polyethylene glycol might also improve circulating half-life $(29,36)$.

The pathways used to deliver RNAs into cells, whether by aptamers or by other delivery methods, remain poorly understood. Our preliminary studies suggest that Cy3-labeled CD4-AsiCs are initially taken up into early endosomes and then escape to the cytosol. Endocytosis might be triggered by activation of the CD4 receptor by aptamer binding or occur via the continuous basal internalization of cell surface receptors. The latter pathway may be more likely, since CD4 cell surface expression is not appreciably altered by CD4-AsiC treatment and since CD4-AsiCs are monomeric and are not expected to crosslink the receptor. Although lack of perturbation of CD4 surface expression would be ideal for using CD4AsiCs as a research tool, a divalent or polyvalent reagent that activates temporary CD4 internalization might also have advantages for HIV prevention or therapy. This might add a third mechanism for inhibiting HIV cellular transmission (removal of the viral receptor from the cell surface) to the other 2 mechanisms demonstrated in the present study (gene silencing and partially blocking the virion binding site on CD4). Once in the endosome, how the RNA is released into the cytosol is unknown. Understanding the uptake and trafficking within cells of CD4-AsiCs will undoubtedly facilitate design improvements.

\section{Methods}

Chimera synthesis. Aptamer-siRNA chimera synthesis was modified from previously described methods $(28,33)$. Primers and template DNA (Supplemental Table 1) were commercially synthesized (IDT). RNA intermediates, transcribed in vitro using Epicentre's DuraScribe kit, were resolved on 15\% dPAGE gels (Invitrogen) and eluted into either buffer A for in vitro studies (1\% $\mathrm{LiClO}_{4}, 7 \mathrm{mM}$ triethylamine; Sigma-Aldrich) or buffer B for in vivo studies (10 mM Tris, pH 7.5-8.0, $50 \mathrm{mM} \mathrm{NaCl}, 1 \mathrm{mM}$ EDTA) prior to ethanol precipitation and desalting using a G25 column (GE). RNAs were mixed in a 1:1 molar ratio with commercially synthesized active siRNA strands (Dharmacon), heated to $90^{\circ} \mathrm{C}$, and allowed to cool slowly to room temperature. In some cases, the active strand was synthesized with a Cy3 label at its 3 ' end.
Vaginal stability assay. $2 \mathrm{nmol}$ CCR5 CD4-AsiC synthesized using 2'-fluoro-pyrimidines, chemically stabilized cholesterol-conjugated CCR5-siRNA, and unmodified 21-mer CCR5 siRNA in $100 \mu$ l PBS were added to $100 \mu \mathrm{l}$ of vaginal fluid obtained from a healthy preovulatory donor. At regular intervals, $20 \mu \mathrm{l}$ was removed, resuspended in TRIzOL reagent (Invitrogen) for RNA extraction, and frozen at $-80^{\circ} \mathrm{C}$ prior to resolution by PAGE analysis 24 hours after the final collection time point. RNA content was analyzed by densitometry, and amounts were calculated relative to RNA content at time 0 .

Cell lines. HeLa, Jurkat, and K562 cells (ATCC) were cultured as previously described $(8,25)$. HeLa-CD4 and HeLa-MAGI CCR5 cell lines, obtained from the NIH AIDS Reagent Program, were maintained in DMEM supplemented with $0.5 \mathrm{mg} / \mathrm{ml} \mathrm{G} 418$. HCT-116 cells (ATCC) were cultured according to the supplier's instructions. Dicer ${ }^{-}-$HCT-116 cells were a gift of B. Vogelstein (Johns Hopkins Medical Institutions, Baltimore, Maryland, USA; ref. 35).

Primary cells. Primary cells from the blood of healthy donors were isolated by Ficoll (GE) density centrifugation and cultured in H10 medium (RPMI 1640 [Cellgro] containing 10\% Human AB Serum [GemCell], 100 U/ml penicillin, and $100 \mu \mathrm{g} / \mathrm{ml}$ streptomycin sulfate). In some cases, $\mathrm{CD}^{+}$cells were separated using immunomagnetic beads (Miltenyi). $\mathrm{CD}^{+} \mathrm{T}$ cells and MDMs were prepared as previously described (8). $\mathrm{CD} 4^{+} \mathrm{T}$ cells were cultured in $\mathrm{H} 10$ containing $60 \mathrm{IU} / \mathrm{ml} \mathrm{IL}-2$ and were activated using $4 \mu \mathrm{g} / \mathrm{ml} \mathrm{PHA}$ (Difco). Resting PBMCs were cultured in $\mathrm{H} 10$ containing $4 \mu \mathrm{g} / \mathrm{ml} \mathrm{IL-15.}$

Viruses. $\mathrm{HIV}_{\mathrm{BaL}}$ and $\mathrm{HIV}_{\mathrm{IIIB}}$ virus were obtained from the NIH AIDS Reagent Program. HIV $\mathrm{BaL}$ was generated by infecting pooled PBMCs that had been stimulated with PHA $(4 \mu \mathrm{g} / \mathrm{ml})$ in $\mathrm{H} 10$ plus $60 \mathrm{IU} / \mathrm{ml} \mathrm{rIL-2}$ (Chiron). $\mathrm{HIV}_{\text {IIIB }}$ was propagated as previously described (47). p24 Ag levels in culture supernatants were measured by HIV-1 p24 Antigen ELISA kit (Perkin Elmer). VSV(G)-pseudotyped HIV-Luc (provided by A. Engelman; DanaFarber Cancer Institute, Boston, Massachusetts, USA) was generated in $239 \mathrm{~T}$ cells as previously described (48). Viral stocks of the HIV-1 $1_{\text {JR-CSF }}$ molecular clone were produced through transfection of HEK293 cells as previously described (49). Supernatant virus was concentrated 1:50 using the PEG-it Virus Precipitation Solution (System Biosciences) per the manufacturer's protocol.

HIV-1 infection in vitro and in situ. Cells were infected with the indicated HIV-1 isolates using an MOI of approximately 1 . Infection of Jurkat and $\mathrm{CD} 4^{+} \mathrm{T}$ cells was by spinoculation at $1,200 \mathrm{~g}$ for 2 hours in the presence of $2 \mathrm{mg} / \mathrm{ml}$ Polybrene. HeLa-CD4 and MDM cells were infected by incubating the cells with virus at $37^{\circ} \mathrm{C}$ for 48 hours. Cells were infected with the single-round VSV(G)-pseudotyped virus for $6-8$ hours at $37^{\circ} \mathrm{C}$, washed, and incubated again at $37^{\circ} \mathrm{C}$ in fresh media for approximately 48 hours prior to analysis as previously described (48). For HIV infection of polarized explants, $\operatorname{HIV}_{\mathrm{BaL}}(\sim 100 \mathrm{ng}$ p 24$)$ was applied to the apical surface of agaroseembedded explants in $200 \mu \mathrm{l}$, and the explants were then incubated at $37^{\circ} \mathrm{C}$. Viral replication in the tissue was assessed by measuring p $24 \mathrm{Ag}$ in the lower transwell chamber using the HIV-1 p24 Antigen ELISA kit (Perkin Elmer).

siRNA transfection. HeLa-CD4 and MDM cells were transfected with Oligofectamine per the manufacturer's protocol. CD4 $4^{+} \mathrm{T}$ cells, Jurkat cells, and K562 cells were transfected by AMAXA according to the manufacturer's protocol (Lonza). See Supplemental Table 2 for all siRNA sequences.

Flow cytometry. Direct immunostaining of CD3, CD4, CD8, CD14, $\mathrm{CD} 45$, and CCR5 was performed using 1:20 dilutions of murine $\mathrm{mAb}$ for 30-60 minutes at $4{ }^{\circ} \mathrm{C}$ (BioLegend). Cells were stained in PBS containing $0.5 \%$ FCS, $1 \mathrm{mM}$ EDTA, and $25 \mathrm{mM}$ HEPES. Samples were washed twice in the same buffer. Data for 1- and 2-color staining experiments were acquired using FACSCalibur (BD Biosciences); for multicolor experiments, data were acquired using FACS-Canto II (BD Biosciences). All data analysis was performed using FlowJo (Treestar Inc.). 
Fluorescence microscopy. Fluorescence microscopy was performed as previously described (24) using primary antibodies (BioLegend) and secondary donkey anti-mouse antibodies (Invitrogen). All images shown were acquired using a $\times 60$ oil objective.

Intracellular 24 staining. Intracellular staining, performed as previously described (8), was analyzed on a FACSCalibur with Cell Quest software (Becton Dickinson) and/or FlowJo software. The p24 MFI was normalized relative to the mock-treated control.

Immunoblot. Total cell extracts were prepared in RIPA buffer $(50 \mathrm{mM}$ Tris-HCl pH 7.4, 150 mM NaCl, 1 mM EDTA, 1\% Triton X-100, 1\% sodium deoxycholate, $0.1 \%$ SDS). Protein concentration was measured using the BCA Protein Assay Reagent (Pierce), and $10 \mu \mathrm{g}$ of total protein was resolved on $10 \%$ SDS-PAGE, transferred to cellulose membranes (Immobilon-P, Millipore), and probed with mouse $\mathrm{mAb}$ against lamin $\mathrm{A}$ (BioLegend). The membranes were developed using SuperSignal West Femto (Pierce). For loading control, $10 \mu \mathrm{g}$ of total protein was resolved on $10 \%$ SDS-PAGE and probed using anti-tubulin mouse $\mathrm{mAb}$ (Sigma-Aldrich).

In vitro Dicer cleavage assay. Recombinant Dicer cleavage assay was performed using the Turbo Dicer siRNA Generation Kit (Genlantis) according to the manufacturer's protocol.

$5^{\prime}$-RACE and sequence analysis. 5'-RACE was performed using the First Choice RLM-RACE Kit (Ambion) according to the manufacturer's protocol, modified to use 45 cycles of amplification for the nested PCR reaction. The nested PCR product was cloned using standard M13 and T7 primers into the PGEM-T Easy vector (Promega) for sequencing at the Dana-Farber Harvard Cancer Center Sequencing Core (Boston, Massachusetts, USA).

Luciferase assay. The luciferase activity of VSV(G)-pseudotyped reporter virus-infected samples was measured 48 hours after transfection using the Luciferase Assay System (Promega) and a Top count NXT microplate reader (Perkin Elmer) per the manufacturer's instructions. Background luminescence was subtracted from all values, and data were normalized relative to mock-treated controls.

qRT-PCR. qRT-PCR was performed as previously described (23) using primers in Supplemental Table 3. mRNA expression was normalized to $G A P D H$, then calculated as a percentage relative to mock-treated controls.

Human cervical polarized tissue explants. Human cervical tissue was obtained from healthy human donors undergoing hysterectomy for benign conditions. Tissue was immediately sectioned into approximately $3-\mathrm{mm}^{3}$ specimens and then oriented with the apical epithelial surface facing up on the membrane of a 12-transwell system (Corning). Explants were then embedded in $3 \%$ agarose as described previously (40), and the integrity of the agarose seal was tested using Trypan blue. The polarized explants were cultured in $200 \mu \mathrm{l} \mathrm{H} 10$ medium. Cy3-labeled AsiCs in $50 \mu \mathrm{l}$ Optimem (Invitrogen) were applied to the apical surface, and the explants were then incubated at $37^{\circ} \mathrm{C}$ for $4-6$ hours before adding $150 \mu \mathrm{l} \mathrm{H} 10$ to each well. The treatment was repeated 24 hours later, then the tissue was incubated for an additional 48 hours at $37^{\circ} \mathrm{C}$ prior to collagenase digestion and analysis. All tissues were obtained anonymously and were considered exempt from informed consent. Approval was obtained from the IRBs of Massachusetts General Hospital and Brighan and Women's Hospital. Human tissues from Beth Israel Deaconess Medical Center were deemed exempt from IRB approval. E. Oliva (Massachusetts General Hospital, Boston, Massachusetts, USA) provided some of the human samples.

Collagenase digestion of human and mouse vaginal tissue. 48 hours after the second application of RNA, specimens were removed from the transwell. 10 polarized explants per treatment condition were pooled and digested in $10 \mathrm{ml}$ RPMI containing $1 \mathrm{mg} / \mathrm{ml}$ collagenase II (Sigma-Aldrich) for 30 minutes at $37^{\circ} \mathrm{C}$ with shaking. Samples were disrupted in a gentleMACS dissociator (Miltenyi) using the C. 01 program for 30 minutes at $37^{\circ} \mathrm{C}$ both before and after collagenase digestion. Cell suspensions were passed through a $70-\mu \mathrm{m}$ cell strainer (BD Falcon), washed with $30 \mathrm{ml}$ RPMI, and stained for flow cytometry.

Hematoxylin and eosin staining. Explants treated for 24 hours with $4 \mu \mathrm{M}$ AsiCs were fixed in $3.7 \%$ formalin (Sigma-Aldrich) and paraffin embedded. $5-\mu \mathrm{m}$ tissue sections were stained with hematoxylin and eosin (ThermoFisher Scientific). Images were acquired using a Zeiss Axiovert 200M microscope and Slidebook software (Intelligent Imaging).

Cytotoxicity assay. Cellular cytotoxicity was quantified by measuring LDH release into the culture medium using the Roche LDH assay according to the manufacturer's instructions. Briefly, $100 \mu \mathrm{l}$ of culture medium harvested 24 hours after treatment was mixed with $100 \mu \mathrm{LDH}$ reaction mix and incubated for 30 minutes at room temperature. Absorbance at $490 \mathrm{~nm}$ was measured using a Spectra MAX 340PC microplate counter (Molecular Devices) and normalized to absorbance at $650 \mathrm{~nm}$. Culture medium from tissue treated with $1 \%$ Triton X-100 (ThermoFisher Scientific) served as a positive control.

IFN and cytokine induction. An equimolar mixture of CD4-AsiC or PSMAAsiC targeting gag and vif (total concentration $4 \mu \mathrm{M}$ ) was applied to the apical surface of the explant, and RNA was extracted using TRIzoL reagent (Invitrogen) 6 and 24 hours later. Treatment with poly(I:C) $(50 \mu \mathrm{g} / \mathrm{ml}$; Invivogen) served as a positive control. qRT-PCR was performed as above (see Supplemental Table 3 for primers). mRNA expression was normalized to $G A P D H$, then normalized relative to mock-treated controls.

In vivo treatment and HIV challenge. All in vivo experiments were performed using immunodeficient mice bearing human bone marrow (either NOD/ SCID or NSG) following reconstitution with CD34+ cells from human fetal liver and surgical human thymic graft (i.e., BLT mice) as previously described (42). Uptake and silencing was assessed in NOD/SCID-BLT mice following 2 IVAG treatments of Cy3-labeled AsiCs, at the indicated doses, 72 hours and 48 hours prior to sacrifice. Vaginal tissue was extracted, and a single-cell suspension was isolated by collagenase digestion and stained as described above. HIV protection was assessed in NSG-BLT mice treated IVAG (a) 48 hours before challenge with $10 \mu \mathrm{l}$ PBS containing 80 pmol CCR5 CD4-AsiCs; (b) 24 hours before challenge with $10 \mu$ l PBS containing 40 pmol each of CCR5, gag, and vif CD4-AsiCs; and (c) 4 hours after challenge with $10 \mu \mathrm{l}$ PBS containing 40 pmol each of gag and vif CD4-AsiCs. Female mice were challenged with atraumatic IVAG instillation of $10^{5}$ $\mathrm{TCID}_{50} \mathrm{HIV}_{\mathrm{JR}-\mathrm{CSF}}$ in $10 \mu \mathrm{l} \mathrm{PBS}$ as previously described (44). Animal work was approved by the Animal Care and Use Committees of Massachusetts General Hospital and Harvard Medical School.

Analysis of HIV infection. Blood was obtained by venipuncture at weekly intervals for 12 weeks after HIV challenge. Cells were pelleted by centrifugation, and plasma was stored at $-80^{\circ} \mathrm{C}$ until analysis. Cell pellets were twice treated with rbc lysis buffer (Sigma-Aldrich), washed with flow cytometry buffer described above, and stained for CD3, CD4, and CD8. Viral RNA was extracted from $75 \mu$ l plasma using the QiaAmp Viral RNA kit (QIAGEN) according to the manufacturer's instructions. cDNA was reverse transcribed using SuperscriptIII (Invitrogen) and HIV-gag-specific primers (Supplemental Figure 2) according to the manufacturer's protocol. qRT-PCR was performed as described above. The remaining serum was aliquoted for p24 Ag ELISA (Perkin Elmer), performed according to the manufacturer's instructions.

Statistics. Data for most experiments were analyzed by Student's $t$ test. All $P$ values are for 2-tailed significance tests. For analysis of data based on independent experiments using samples from multiple donors, 1-way ANOVA with Dunnett multiple-comparison test was performed using GraphPad Prism (GraphPad Software). Assessment of HIV infection was by 2 -way ANOVA with Dunnett multiple-comparison test. $P$ values less than 0.05 were considered significant. The limit of detection was calculated using a previously described method (50) and is shown as the average of the calculated limit of detection for each individual assay. 


\section{Acknowledgments}

This work was supported by the NIAID (NIH grants AI070302 and AI090671), Massachusetts Life Sciences Center (to J. Lieberman), Harvard CFAR and Ragon Institute (to J. Lieberman, A.D. Luster, and A.M. Tager), predoctoral fellowships from the Cancer Research Institute and the Ryan Foundation (to L.A. Wheeler), a Point Foundation scholarship (to L.A. Wheeler), and a Harvard CFAR Scholar award (to R. Trifonova). We thank members of the Lieberman laboratory, N. Barteneva, K. Ketman, M. Manoharan, K. Charisse, C.M. Boutwell, S. Biswas, and P.T.N. Sarkis for scientific advice; E. Oliva for providing human tissue samples; H.S. Shin and S. Brooks for technical assistance; and the NIH AIDS Reagent Program, A. Engelman, and B. Vogelstein for reagents.

Received for publication November 22, 2010, and accepted in revised form March 30, 2011.

Address correspondence to: Judy Lieberman, 200 Longwood Avenue WAB 255, Boston, Massachusetts 02115, USA. Phone: 617.713.8600; Fax: 617.713.8620; E-mail: lieberman@idi.harvard.edu.
1. Abdool Karim Q, et al. Effectiveness and safety of tenofovir gel, an antiretroviral microbicide, for the prevention of HIV infection in women. Science. 2010;329(5996):1168-1174.

2. Grant RM, et al. Whither or wither microbicides? Science. 2008;321(5888):532-534.

3. Novina CD, et al. siRNA-directed inhibition of HIV-1 infection. Nat Med. 2002;8(7):681-686.

4. CapodiciJ, Kariko K, Weissman D. Inhibition of HIV-1 infection by small interfering RNA-mediated RNA interference. J Immunol. 2002;169(9):5196-5201.

5. Jacque JM, Triques K, Stevenson M. Modulation of HIV-1 replication by RNA interference. Nature. 2002;418(6896):435-438.

6. Lee NS, et al. Expression of small interfering RNAs targeted against HIV-1 rev transcripts in human cells. Nat Biotechnol. 2002;20(5):500-505.

7. Coburn GA, Cullen BR. Potent and specific inhibition of human immunodeficiency virus type 1 replication by RNA interference. J Virol. 2002; 76(18):9225-9231.

8. Song E, et al. Sustained small interfering RNAmediated human immunodeficiency virus type 1 inhibition in primary macrophages. J Virol. 2003; 77(13):7174-7181

9. Lee SK, et al. Lentiviral delivery of short hairpin RNAs protects CD4 $\mathrm{T}$ cells from multiple clades and primary isolates of HIV. Blood. 2005; 106(3):818-826.

10. van't Wout AB, et al. Macrophage-tropic variants initiate human immunodeficiency virus type 1 infection after sexual, parenteral, and vertical transmission. J Clin Invest. 1994;94(5):2060-2067.

11. Margolis L, Shattock R. Selective transmission of CCR5-utilizing HIV-1: the 'gatekeeper' problem resolved? Nat Rev Microbiol. 2006;4(4):312-317.

12. Mills SG, DeMartino JA. Chemokine receptordirected agents as novel anti-HIV-1 therapies. Curr Top Med Chem. 2004;4(10):1017-1033.

13. Lederman MM, et al. Prevention of vaginal SHIV transmission in rhesus macaques through inhibition of CCR5. Science. 2004;306(5695):485-487.

14. Gulick RM, et al. Phase 2 study of the safety and efficacy of vicriviroc, a CCR5 inhibitor, in HIV-1-Infected, treatment-experienced patients: AIDS clinical trials group 5211. J Infect Dis. 2007; 196(2):304-312.

15. Gulick RM, et al. Maraviroc for previously treated patients with R5 HIV-1 infection. NEnglJMed. 2008; 359(14):1429-1441.

16. Fatkenheuer G, et al. Subgroup analyses of maraviroc in previously treated R5 HIV-1 infection. NEngl JMed. 2008;359(14):1442-1455.

17. Kuritzkes DR. HIV-1 entry inhibitors: an overview. Curr Opin HIV AIDS. 2009;4(2):82-87.

18. Dean M, et al. Genetic restriction of HIV-1 infection and progression to AIDS by a deletion allele of the CKR5 structural gene. Hemophilia Growth and Development Study, Multicenter AIDS Cohort
Study, Multicenter Hemophilia Cohort Study, San Francisco City Cohort, ALIVE Study. Science. 1996;273(5283):1856-1862.

19. Samson M, et al. Resistance to HIV-1 infection in caucasian individuals bearing mutant alleles of the CCR-5 chemokine receptor gene. Nature. 1996;382(6593):722-725.

20. Huang Y, et al. The role of a mutant CCR5 allele in HIV-1 transmission and disease progression. Nat Med. 1996;2(11):1240-1243.

21. Zimmerman PA, et al. Inherited resistance to HIV-1 conferred by an inactivating mutation in $\mathrm{CC}$ chemokine receptor 5 : studies in populations with contrasting clinical phenotypes, defined racial background, and quantified risk. Mol Med. 1997;3(1):23-36.

22. Galvani AP, Novembre J. The evolutionary history of the CCR5-Delta32 HIV-resistance mutation. Microbes Infect. 2005;7(2):302-309.

23. Palliser $D$, et al. An siRNA-based microbicide protects mice from lethal herpes simplex virus 2 infection. Nature. 2006;439(7072):89-94.

24. Wu Y, et al. Durable protection from Herpes Simplex Virus-2 transmission following intravaginal application of siRNAs targeting both a viral and host gene. Cell Host Microbe. 2009;5(1):84-94.

25 . Song E, et al. Antibody mediated in vivo delivery of small interfering RNAs via cell-surface receptors. Nat Biotechnol. 2005;23(6):709-717.

26. Peer D, Zhu P, Carman CV, Lieberman J, Shimaoka $M$. Selective gene silencing in activated leukocytes by targeting siRNAs to the integrin lymphocyte function-associated antigen-1. Proc Natl Acad Sci U S A. 2007;104(10):4095-4100.

27. Kumar P, et al. T cell-specific siRNA delivery suppresses HIV-1 infection in humanized mice. Cell. 2008;134(4):577-586

28. McNamara JO 2nd, et al. Cell type-specific delivery of siRNAs with aptamer-siRNA chimeras. Nat Biotechnol. 2006;24(8):1005-1015.

29. Dassie JP, et al. Systemic administration of optimized aptamer-siRNA chimeras promotes regression of PSMA-expressing tumors. Nat Biotechnol. 2009;27(9):839-849.

30. Zhou J, Li H, Li S, Zaia J, Rossi JJ. Novel dual inhibitory function aptamer-siRNA delivery system for HIV-1 therapy. Mol Ther. 2008;16(8):1481-1489.

31. Zhou J, et al. Selection, characterization and application of new RNA HIV gp 120 aptamers for facile delivery of Dicer substrate siRNAs into HIV infected cells. Nucleic Acids Res. 2009;37(9):3094-3109.

32. Neff CP, et al. An aptamer-siRNA chimera suppresses HIV-1 viral loads and protects from helper CD4(+) T cell decline in humanized mice. Sci Transl Med. 2011;3(66):66ra66.

33. Davis KA, Lin Y, Abrams B, Jayasena SD. Staining of cell surface human CD4 with 2'-F-pyrimidinecontaining RNA aptamers for flow cytometry. Nucleic Acids Res. 1998;26(17):3915-3924.
34. Shultz LD, Ishikawa F, Greiner DL. Humanized mice in translational biomedical research. Nat Rev Immunol. 2007;7(2):118-130.

35. Cummins JM, et al. The colorectal microRNAome. Proc Natl Acad Sci U S A. 2006;103(10):3687-3692.

36 . Soutschek J, et al. Therapeutic silencing of an endogenous gene by systemic administration of modified siRNAs. Nature. 2004;432(7014):173-178.

37. Elbashir SM, Lendeckel W, Tuschl T. RNA interference is mediated by 21 - and 22-nucleotide RNAs. Genes Dev. 2001;15(2):188-200.

38. Arthos J, et al. Identification of the residues in human CD4 critical for the binding of HIV. Cell. 1989;57(3):469-481.

39. Greenhead P, Hayes P, Watts PS, Laing KG, Griffin GE, Shattock RJ. Parameters of human immunodeficiency virus infection of human cervical tissue and inhibition by vaginal virucides. J Virol. 2000;74(12):5577-5586.

40. Collins KB, Patterson BK, Naus GJ, Landers DV, Gupta P. Development of an in vitro organ culture model to study transmission of HIV-1 in the female genital tract. Nat Med. 2000;6(4):475-479.

41. Robbins M,Judge A, MacLachlan I. siRNA and innate immunity. Oligonucleotides. 2009;19(2):89-102.

42. Brainard DM, et al. Induction of robust cellular and humoral virus-specific adaptive immune responses in human immunodeficiency virus-infected humanized BLT mice. J Virol. 2009;83(14):7305-7321.

43. Berges BK, Akkina SR, Folkvord JM, Connick E, Akkina R. Mucosal transmission of R5 and $\mathrm{X} 4$ tropic HIV-1 via vaginal and rectal routes in humanized Rag2//- gammac $^{-/-}$(RAG-hu) mice. Virology. 2008;373(2):342-351.

44. Denton PW, et al. Antiretroviral pre-exposure prophylaxis prevents vaginal transmission of HIV- 1 in humanized BLT mice. PLoS Med. 2008;5(1):e16.

45. Peer D, Park EJ, Morishita Y, Carman CV, Shimaoka M. Systemic leukocyte-directed siRNA delivery revealing cyclin D1 as an anti-inflammatory target. Science. 2008;319(5863):627-630.

46. Haase AT. Targeting early infection to prevent HIV-1 mucosal transmission. Nature. 2010; 464(7286):217-223

47. Brass AL, et al. Identification of host proteins required for HIV infection through a functional genomic screen. Science. 2008;319(5865):921-926.

48. Shun MC, et al. LEDGF/p75 functions downstream from preintegration complex formation to effect gene-specific HIV-1 integration. Genes Dev. 2007;21(14):1767-1778.

49. Boutwell CL, Rowley CF, Essex M. Reduced viral replication capacity of human immunodeficiency virus type 1 subtype $C$ caused by cytotoxic-T-lymphocyte escape mutations in HLA-B57 epitopes of capsid protein. J Virol. 2009;83(6):2460-2468.

50. Armbruster DA, Pry T. Limit of blank, limit of detection and limit of quantitation. Clin Biochem Rev. 2008;29(suppl 1):S49-S52. 